\title{
Electromagnetic proton form factors: perspectives for PANDA
}

\author{
Egle Tomasi-Gustafsson ${ }^{1,2, a}$ and Alaa Dbeyssi ${ }^{2}$, on behalf of the PANDA Collaboration \\ ${ }^{1}$ CEA,IRFU,SPhN, Saclay, 91191 Gif-sur-Yvette, France \\ ${ }^{2}$ Univ Paris-Sud, CNRS/IN2P3, IPNO, UMR 8608, 91406 Orsay, France
}

\begin{abstract}
The PANDA collaboration studies fundamental aspects of the strong interaction in the transition region between non-perturbative and perturbative QCD, investigating charmonium spectroscopy, hybrids and glueballs, hypernuclei, light and heavy meson production with antiproton beams. In this contribution we focus on leptonic final channels which give access to nucleon electromagnetic form factors. The expected precision on the electric and magnetic form factors of the proton in the time-like region and the radiative corrections to be applied to the data are discussed.
\end{abstract}

\section{Introduction}

The PANDA experiment (antiProton ANnichilation at DArmstadt) is one of the four main experiments at the Facility for Antiproton and Ion Research (FAIR) [1]. FAIR is under construction on the area of GSI, in Darmstadt (Germany) and it is intended to gather a large community of 2500 physicists interested in hadron and nuclei structure, in nuclear matter, in plasma and atomic physics, around the main experiments: PANDA, CBM, NUSTAR, and APPA. FAIR will offer high quality beams of protons, antiprotons, nuclei in the intermediate energy range as well as installations for applied and atomic physics. The PANDA collaboration with more than 500 scientists from 17 countries intends to do basic research on aspects of the manifestation of the weak and strong forces, exotic states of matter and the structure of hadrons. A high intensity antiproton beam of momentum in the range 1.5-15 $\mathrm{GeV} / \mathrm{c}$ will collide with a fixed target $(p, d$, nuclei...) inside the PANDA detector, with a luminosity of $10^{32} \mathrm{~cm}^{-2} \mathrm{~s}^{-1}$ corresponding to one order of magnitude larger than previously achieved.

In order to gather all the necessary information from the antiproton-proton collisions, the PANDA detector will be able to provide precise trajectory reconstruction, energy and momentum measurements and very efficient identification of charged particles and photons. For the specific interest of the present contribution, this will allow to measure the proton-antiproton annihilation in two leptons, selecting the interesting events from the huge hadronic background.

It is well known that, assuming that the annihilation occurs via one virtual photon of mass $q^{2}$, such reaction allows to access the electric and magnetic form factors of the proton, in the time-like region of the transferred momenta. The detection of an accompanying pion will allow to investigate for the first time the "unphysical region", below the kinematical threshold, $q^{2}=4 m^{2}, m$ being the proton mass.

\footnotetext{
ae-mail: tomasi@ipno.in2p3.fr
} 
The precision that can be achieved nowadays allows to think in terms of a global view of FFs, in the whole kinematical region and to focus on the theoretical approaches which can give an overall description in space-like(time-like) which is accessible through scattering(annihilation) processes. The comparison of the data, including polarized electron-proton elastic scattering on one side, and electron positron annihilation into proton antiproton with initial state radiation on the other side, shows peculiar features: -time-like proton FFs are twice as large as in space-like at comparable $q^{2}$, - the behavior of the electric and magnetic distributions as functions of the transferred momentum are different, - protons seem to behave as point-like particles at threshold. We present here the status of the feasibility studies of the annihilation reaction $\bar{p}+p \rightarrow e^{+}+e^{-}$at PANDA, with specific interest in two aspects: event selection and radiative corrections.

\section{Simulation}

Assuming one photon exchange, the differential cross section for the annihilation process $\bar{p} p \rightarrow e^{+} e^{-}$ in the CM system is expressed as a function of the proton electric $\left(G_{E}\right)$ and magnetic $\left(G_{M}\right)$ FFs as [2]:

$$
\frac{d \sigma}{d c}=\mathcal{N} \mathcal{D}, \mathcal{N}=\frac{\pi \alpha^{2}}{2 \beta s}, \beta=\sqrt{1-\frac{1}{\tau}}, \tau=\frac{s}{4 m^{2}}, s=q^{2}, \mathcal{D}=\left(1+c^{2}\right)\left|G_{M}\right|^{2}+\frac{1}{\tau} \sin ^{2} \theta\left|G_{E}\right|^{2}
$$

where $c=\cos \theta, \theta$ is the $\mathrm{CM}$ electron angle, $\alpha$ is the electromagnetic coupling constant and $m$ is the proton mass. Eq. 1 can also be written as a function of the angular asymmetry $\mathcal{A}$, defined as the slope of the linear $c^{2}$ dependence, as:

$$
\frac{d \sigma}{d c}=\sigma_{0}\left[1+\mathcal{A} c^{2}\right], \sigma_{0}=\mathcal{N}\left(\left|G_{M}\right|^{2}+\frac{1}{\tau}\left|G_{E}\right|^{2}\right), \mathcal{A}=\frac{\tau\left|G_{M}\right|^{2}-\left|G_{E}\right|^{2}}{\tau\left|G_{M}\right|^{2}+\left|G_{E}\right|^{2}}=\frac{\tau-R^{2}}{\tau+R^{2}}, R=\left|G_{E}\right| /\left|G_{M}\right|
$$

where $\sigma_{0}$ is the value of the differential cross section at $\theta=\pi / 2$.

\subsection{Event selection}

We have considered three values of the transferred momentum $q^{2}=5.4,8.2,13.9 \mathrm{GeV}^{2}$. According to the phase space generator, $\sim 10^{6}$ Monte Carlo events have been generated for the signal and $\sim 10^{8}$ events for the main background $\bar{p} p \rightarrow \pi^{+} \pi^{-}$. Events for the very forward and the very backward angles are lost due to the acceptance of the detector. The present analysis is limited to the angular range $c=[-0.8,0.8]$. The PANDAGrid was used to process all the events (simulation and analysis). The handling of such a huge amount of data $\left[3 \times\left(10^{8}+10^{6}\right)\right]$ was taken in charge by the Vienna group and required specific optimizations at the level of the job submission and the PANDAGrid management. The output in form of n-tuples were also stored in Orsay for further analysis. The background events were completely handled at the level of the PANDAGrid. The number of running jobs in parallel increased from typically 100 at the beginning of the production to more than 800 at the end. This is due to the fact that new sites became operational during that period and to the optimization of the procedures. The achieved production speed was $6 \times 10^{6}$ events/day [3].

The simulations using the official software of the experiment, PANDARoot, consists of five steps. The generated particles propagate through the detector using either GEANT3 (this study) or GEANT4. The information on the hits and the energy losses is digitized, including the electronic noise, into a response of the different detectors. The physical quantities such as momentum, ratio of energy loss along the path length in each straw tube $(d E / d x)$, Cerenkov angle in the DIRC detector, and energy deposit in the electromagnetic calorimeter are reconstructed for particle identification. The 

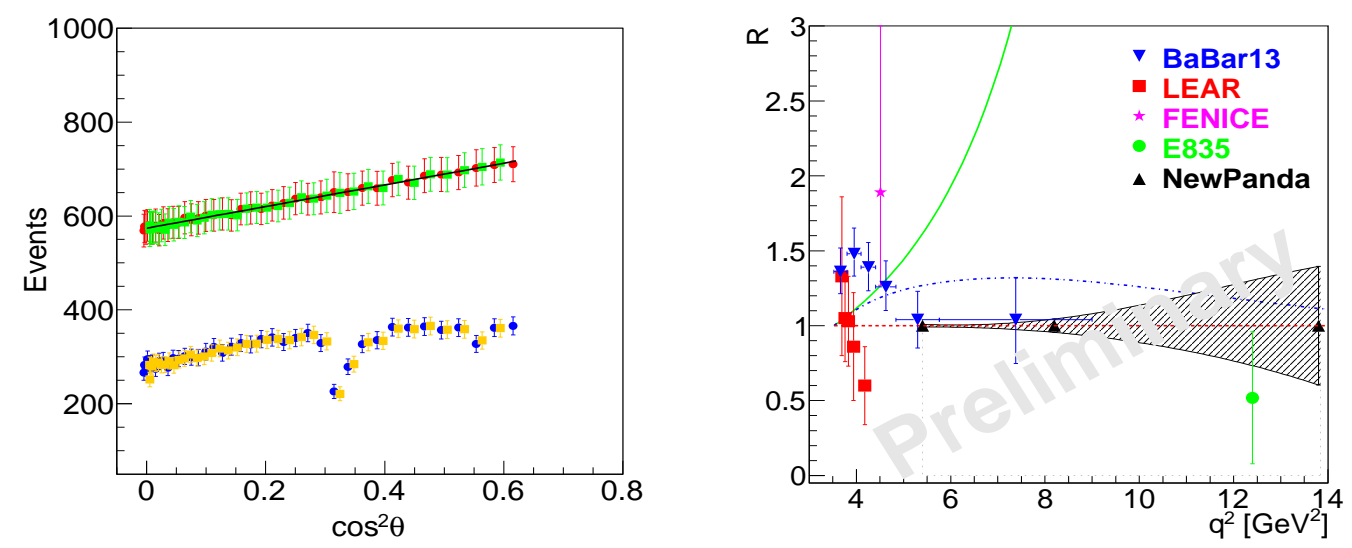

Figure 1. (Left) Monte Carlo events (blue-forward, yellow-backward) renormalized to physical events and corrected for efficiency (red-forward, green-backward), and linear fit (black line) as a function of $c^{2}$, for $q^{2}=8.2$ $\mathrm{GeV}^{2}$. (Right) Expected statistical precision on the determination of the proton FF ratio for $R=1$ (magenta triangles) as a function of $q^{2}$, compared with the existing data and with theoretical predictions (see also [4]).

probabilities to identify a given particle as an electron, a muon, a pion, a kaon or a proton are then calculated for each detector and a global particle identification (PID) likelihood is calculated. The last step is the analysis of the reconstructed events in order to identify the signal from the background and to extract physical quantities.

The electron reconstructed events, after applying the kinematical and the PID cuts, are used for the extraction of the proton FFs. The angular distributions for the electron, unifom in phase space, have to be rescaled using a weight of the form $\omega=1+\mathcal{A} c^{2}$ (physical events). The angular asymmetry $\mathcal{A}(s)$ is given according to $R=\left|G_{E}\right| /\left|G_{M}\right|=1$. We did a two parameter fit, determining the angular asymmetry and the cross section at $90^{\circ}$. The obtained angular distribution in $c^{2}$ has been fitted with a straight line (Fig. 1 left). The three points are plotted on the $R=1$ axis. The error for the last point is comparable to the highest experimental point (Fig. 1 right). Compared to the previous work [4], the present simulation performed within the PANDAGrid relies on a more realistic description of the detector as well as improved algorithms for tracking and selection. The obtained efficiency is larger than $40 \%$ in the considered $q^{2}$ range, suppressing each of the $10^{8}$ pions. The efficiency depends on the cuts used to eliminate the pions and the final cuts will be set on real data.

\subsection{Radiative Corrections}

The size of radiative corrections is also strongly related to the experimental cuts. Radiative corrections affect not only the absolute value but also the dependence of the observables on the relevant kinematical variables. In the present case, they have been calculated at first order in [5], and at all orders, but in the leading logarithm approximation in Refs $[5,6]$.

First order calculations take into account the initial and final state emission, as well as their interference, virtual corrections (absorption and emission of photons from the electron and proton lines, two photon exchange and vacuum polarization. They can be divided in odd and even contributions 
with respect to $c$. Even contribution do not affect the slope of the $c^{2}$ dependence of the cross section and acts as a global normalization. The corrections from Ref. [5] are shown in Fig. 2.

The results given by the simulation, which are based on PHOTOS [6] are consistent with the analytical calculations for what concerns the even contributions, except for the vacuum polarization (not included). Even contributions do not affect the form factor ratio. Odd contributions arising from the interference between the initial and the final state and, at a lesser extent, the two photon contribution, are not calculated by PHOTOS. The odd contribution results in a distortion of the angular distribution which may affect the experimental result. The implementation of the missing contribution in PHOTOS or the development of a dedicated generator is under discussion.

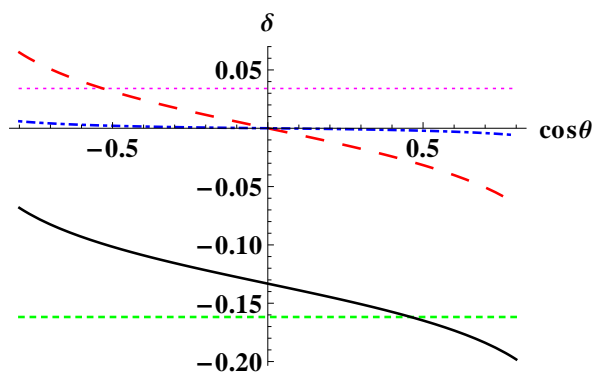

Figure 2. Radiative corrections factor $\delta$, as function of the cosine of the electron emission angle, according to Ref. [5] for $\mathrm{s}=12.8 \mathrm{GeV}^{2}$ and $E_{\gamma}^{\max }=100 \mathrm{MeV}$ : vacuum excitation (magenta, dotted), odd contribution mainly from soft photon emission (red, long dashed) and from two photon exchange (blue, dot-dashed), even contribution (green, dashed), total (black,solid). The total correction is represented by the black solid line.

\section{Conclusions}

After a brief introduction on the FAIR facility and on the PANDA experiment, we have presented the results of a simulation dedicated to the measurement of the time-like electromagnetic form factors of the proton, based on the PANDARoot software and using the PANDAGrid. The efficiency of such a system allowed to perform a large scale simulation of the reactions $\bar{p}+p \rightarrow e^{+}+e^{-}$and $\bar{p}+p \rightarrow \pi^{+}+\pi^{-}$ which is the main background. $10^{6}$ events $\left(10^{8}\right.$ for the background) for each of the three kinematical points have been simulated and analyzed for $q^{2}=5.4,8.4$ and $13.9 \mathrm{GeV}^{2}$. We have shown that the form factor ratio can be measured with a reasonable accuracy up to $q^{2} \simeq 14 \mathrm{GeV}^{2}$. Radiative corrections have to be taken into account: the necessity to include odd contributions and high orders will depend on the resolution and on the errors inherent to the measurements. PANDA will bring a precise knowledge of the proton form factor ratio in a large kinematical range, together with the cross section measurement of $\bar{p}+p \rightarrow e^{+}+e^{-}$up to $\simeq 30 \mathrm{GeV}^{2}$, in frame of a global effort with other world facilities as electron positron colliders (BES, Novosibirsk) and electron accelerators (JLab), in order to progress in the knowledge of the internal structure of the proton in the non-perturbative domain of QCD.

Thanks are due to the PANDA IPN group, in particular to D. Marchand and B. Ramstein, for careful reading and useful remarks.

\section{References}

[1] The PANDA Collaboration, arXiv:hep-ex0903.3905 (2009)

[2] A. Zichichi, S. Berman, N. Cabibbo, R. Gatto, Nuovo Cim. 24, 170 (1962)

[3] P. Buehler, private communication

[4] M. Sudol, et al., Eur.Phys.J. A44, 373 (2010)

[5] A. Ahmadov, V. Bytev, E. Kuraev, E. Tomasi-Gustafsson, Phys.Rev. D82, 094016 (2010)

[6] P. Golonka, Z. Was, Eur.Phys.J.C 45, 97 (2006); A. Dbeyssi, PANDA Note AD12-121213 